\title{
Atención del dolor agudo como variable de calidad durante la hospitalización
}

\author{
Rocío Mendoza-Trujillo ${ }^{1 *}$, Evelyn Manzano-Victoria ${ }^{1}$, Amparo Cahue-Leal ${ }^{2}$, \\ Monserrat Ma. del Consuelo Huerta ${ }^{3}$ y J. Antonio Álvarez-Canales ${ }^{3}$ \\ ${ }^{1}$ Servicio de Anestesiología; ${ }^{2}$ Servicio de Enfermería; ${ }^{3}$ Comisión Coordinadora de Institutos Nacionales de Salud, ${ }^{4}$ Investigación. Hospital Regional de Alta Especialidad \\ del Bajío, León, Gto., México
}

\begin{abstract}
RESUMEN
Objetivo: Es indispensable que las instituciones de salud y quienes laboran en ellas reconozcan el derecho de los pacientes a recibir la mejor atención del dolor posible, así como que se anticipen al dolor generado por los cuidados de la salud. Metodología: En el Hospital Regional de Alta Especialidad del Bajío se realizó la auditoría clínica en el proceso de atención del dolor como variable de calidad de la atención y de una nueva cultura organizacional, mediante una auditoría clínica que se llevó a cabo durante dos años. Resultados: La auditoría clínica se implementó del 2016 al 2018, periodo en el cual se vio un incremento significativo en el apego a las escalas de evaluación del dolor postoperatorio estandarizadas, del 10 al $48 \%$ en el área médica y hasta del $68 \%$ en el área de enfermería. A su vez, se promovió el diseño de la analgesia perioperatoria multimodal, con una población mayor a 2,500 pacientes atendidos anualmente en la Unidad de dolor agudo. Con ello, el nivel de satisfacción en el usuario se calificó como bueno a excelente en el 90\% de los encuestados. Conclusiones: La auditoría permitió evaluar el dolor del paciente hospitalizado cumpliendo los estándares de calidad para la certificación hospitalaria, diseñar el proceso de atención individualizado a nuestra población de mayor impacto y vincular los servicios hospitalarios involucrados. Los beneficios obtenidos de la presente auditoría pueden ser la base para la formación de un comité clínico de atención al dolor que asegure políticas, permanencia y seguimiento del proceso.
\end{abstract}

Palabras clave: Dolor agudo. Dolor postoperatorio. Evaluación del dolor. Calidad.

\section{ABSTRACT}

Background: Currently, it is imperative for health institutions and their staff to recognize the right of patients to receive the best possible care, as well as anticipate to the pain generated by health care procedures. Methods: In the High Specialty Regional Hospital of Bajío, a clinical audit was conducted on the process of treating pain as a variable of quality of health care and of a new organizational culture, through a project in 3 phases for 2 years. Results: The

\section{Correspondencia:}

${ }^{*}$ Rocío del C. Mendoza-Trujillo

Hospital Regional de Alta Especialidad del Bajío

Blvd. Milenio, 130

Col. San Carlos La Roncha

Date of reception: 24-09-2018

C.P. 37660, León, Gto., México

E-mail: rociomendozat@yahoo.es

Date of acceptance: 16-11-2018

DOI: 10.24875/HMCM.18000160 
clinical audit was implemented from 2016 to 2018, a period in which there was a significant increase in adherence to standardized post-operative pain assessment scales, from $10 \%$ to $48 \%$ in the medical area, and up to $68 \%$ in the nursing area. At the same time, multimodal peri-operative analgesia was promoted, with a population of more than 2,500 patients treated annually in the acute pain unit. After this period, the level of user satisfaction was rated as good to excellent in $90 \%$ of those surveyed. Conclusions: The audit allowed to evaluate and treat pain in the hospitalized post-operated patient by complying with quality standards for hospital certification, to design the process of individualized care for our patient population and to link the hospital departments involved. The benefits obtained from the audit can be the basis for the development of a clinical committee for analgesic care, which ensures policies, permanence and follow-up of the process. (Hosp Med Clin Manag. 2018;11:167-77)

Corresponding author: Rocío del C. Mendoza-Trujillo, rociomendozat@yahoo.es

Key words: Acute pain. Postoperative pain. Pain measurement. Healthcare quality.

\section{INTRODUCCIÓN}

En el mundo, en los últimos 30 años la atención del dolor ha sido protagonista de múltiples foros, tanto médicos como de asociaciones civiles y gubernamentales. En 1990 el consenso era que el dolor debería ser considerado en la atención médica y recibir una evaluación más objetiva y, por ello, se deberían adquirir los conocimientos y fomentarse la investigación necesaria para tratarlo en todas sus vertientes ${ }^{1}$. En el año 1997 en Inglaterra se encuestaron pacientes hospitalizados en 36 instituciones diferentes; un $87 \%$ de ellos decían haber cursado con dolor moderado a severo y solo un $42 \%$ tuvo alivio con el tratamiento instaurado². Estudios en otras partes del mundo reportaron resultados muy similares.

En los siguientes años la Asociación Americana del Dolor (APS, American Pain Society) y la Comisión Conjunta de Acreditación de Organizaciones de Salud (JCAHO) desarrollaron estándares de calidad para el alivio del dolor. Se sugirieron escalas para su evaluación, con énfasis en la estadística y con la necesidad de evitar el uso de las siglas PRN (por razón necesaria) cuando se tratara de prescribir analgésicos; además, se establecieron protocolos adecuados para dosis de rescate o escalar hacia analgésicos de mayor potencia, como los opioides. De manera paralela, se inició la campaña de registrar el dolor como la quinta constante vital $^{3}$.

Las consecuencias de un pobre tratamiento del dolor son multidimensionales. Van desde el incremento en días de estancia hospitalaria y morbilidad hasta una percepción negativa del usuario respecto de su experiencia dentro de un hospital; además, se ha considerado una práctica poco ética, poco empática e, incluso, como violatoria a los derechos humanos por la repercusión del dolor per se y sus consecuencias en la calidad de vida ${ }^{4,5}$. Una consecuencia más tangible es el dolor postoperatorio persistente, aquel dolor agudo asociado a un procedimiento quirúrgico, tratado inadecuadamente y que persiste después de tres meses. El mal manejo se considera causa de cronicidad del dolor y está relacionado con las consecuencias del uso crónico de opioides, con una incidencia de hasta 40,000 casos anuales en Inglaterra, lo que representa hasta el $5 \%$ de las cirugías realizadas 6,7 .

Sin embargo, 20 años después de múltiples acciones, la prevalencia del dolor agudo se reportaba muy similar: para el año 2013 México reportó la presencia de hasta el $80 \%$ de dolor moderado a severo en pacientes hospitalizados y en España, en 2015, entre el 30 y el $70 \%$. 9 .

Por lo anterior, se comenzó a cuestionar la validez de algunas recomendaciones y a actualizar otras, sobre todo aquellas relacionadas con prescripción de opioides. Así mismo, el concepto de analgesia preventiva tomó relevancia: no era prescribir mucho, sino optimizar los esquemas analgésicos; anticiparse al dolor reducía las dosis de analgésicos al mínimo y también sus efectos adversos ${ }^{10}$.

De acuerdo con el contexto mencionado, en los Hospitales Regionales de Alta Especialidad (HRAE) dependientes de la Secretaría de Salud federal en México se detectó la necesidad de obtener estadísticas de la población local que definieran el problema de la evaluación y tratamiento del dolor postoperatorio para introducir las recomendaciones de buena práctica al respecto. En el Hospital Regional de Alta Especialidad del Bajío (HRAEB) se optó por la metodología de la 
auditoría clínica para estandarizar la atención al dolor centrada en el paciente hospitalizado.

\section{MÉTODOS}

La auditoría se realizó de 2016 a 2018 en un proceso continuo, llevado a cabo en tres fases. Dentro de la primera fase de esta auditoría se sensibilizó a los Servicios de anestesiología, cirugía y enfermería en relación a los protocolos y a las buenas prácticas de control del dolor en pacientes postoperados. En la siguiente fase, se estableció el diagnóstico situacional que mostró las necesidades mediatas en la población del HRAEB y los procesos necesarios que implementar para mejorar la calidad en la atención del dolor. En la fase final se realizó la medición de la satisfacción del usuario.

\section{Planteamiento del problema: propósitos de la auditoría}

- Implementar el proceso de atención de dolor agudo postoperatorio en pacientes hospitalizados en el HRAEB mediante la instalación permanente de la auditoría clínica encaminada a la formación y sensibilización de todos los profesionales involucrados en la prevención, diagnóstico y tratamiento del dolor postoperatorio.

- Estandarizar la evaluación del dolor agudo en hospitalización y poblaciones especiales como pacientes sedados, niños que no verbalizan y neonatos.

\section{Definición de estándares}

El proceso de atención al dolor en el HRAEB fue redactado por la coordinación de calidad, la gestión del cuidado de enfermería y la jefatura de anestesiología y algología, con base en las recomendaciones nacionales (Sistema Nacional de Certificación del Consejo Nacional de Salud ${ }^{11}$ ) e internacionales ${ }^{12}$ (Guías de Práctica Cínica de la Asociación Americana del Dolor):

\section{Modelo para certificación, recomendaciones ${ }^{11}$}

- Evaluación de los pacientes (AOP, assesment of patient). El dolor se evalúa en el primer contacto, una vez por turno y de acuerdo a las necesidades del paciente; se usa una herramienta estandarizada y se documenta.

- Derechos del paciente y su familia. La organización respeta y respalda el derecho del paciente a obtener la evaluación y el tratamiento del dolor adecuados.

- Atención de pacientes (COP, care of patients). La organización define un proceso para la atención del dolor con base en la planificación de la atención, incluida la identificación de consideraciones especiales. Debe contarse con la documentación necesaria (formato de registro) para que el equipo de atención trabaje y se comunique de manera efectiva. Es indispensable el consentimiento informado y debe realizarse la monitorización del paciente durante el proceso de atención (evaluación del dolor). El personal involucrado en el proceso de atención debe contar con las competencias, insumos y equipo especializados necesarios para llevar a cabo su labor cabalmente. Finalmente, debe contemplarse la comunicación y educación de los pacientes y sus familiares respecto al tratamiento del dolor en el contexto de sus creencias personales, culturales y religiosas. Todo esto será evaluado mediante la realización de encuestas dirigidas a los pacientes y familiares.

\section{Guías de práctica clínica para el dolor postoperatorio de la Sociedad Americana del Dolor ${ }^{12}$}

En el año 2015 la APS aprobó 30 recomendaciones, de las cuales se consideraron dos para la redacción de esta auditoría y el algoritmo de atención producto de esta, con enfoque en la estandarización en la evaluación del dolor: la recomendación 5, que hace énfasis en el uso de una herramienta validada para evaluación, seguimiento y ajustes de la terapéutica utilizada; y la recomendación 6 , que promueve técnicas de analgesia multimodal para el tratamiento del dolor postoperatorio como parte del diseño de la analgesia.

\section{Desarrollo de una escala unificada de evaluación del dolor para utilizarse en la auditoría}

Se desarrolló una escala unificada de evaluación del dolor para utilizarse en la auditoría, denominada en lo sucesivo, y solo para efectos de esta publicación, 
Tabla 1. Escalas validadas para la evaluación del dolor

\begin{tabular}{|c|c|c|}
\hline Abreviatura & $\begin{array}{l}\text { Nombre de la } \\
\text { escala }\end{array}$ & Valores \\
\hline EN & Escala numérica & $\begin{array}{l}\text { Otorga una puntuación } 11 \text { puntos } \\
\text { del } 0 \text { al } 10 \text {, donde } 0 \text { es nada y } 10 \\
\text { es el máximo dolor } \\
\text { experimentado }\end{array}$ \\
\hline EVERA & $\begin{array}{l}\text { Escala verbal } \\
\text { análoga }\end{array}$ & $\begin{array}{l}4 \text { puntos: nada, leve, moderado } 0 \\
\text { severo }\end{array}$ \\
\hline $\mathrm{EF}$ & Escala facial & $\begin{array}{l}\text { Señala la imagen de la expresión } \\
\text { que corresponde al dolor } \\
\text { percibido }\end{array}$ \\
\hline EVA & $\begin{array}{l}\text { Escala visual } \\
\text { analógica }\end{array}$ & $\begin{array}{l}\text { Señala de una línea de } 0 \text { a } 10 \\
\text { con color azul a rojo } \\
\text { (termómetro) }\end{array}$ \\
\hline
\end{tabular}

Adaptada de Chou, et al., $2016^{12}$.

escala del HRAEB. La escala constaba de cuatro escalas unidimensionales: escala visual analógica (EVA), escala numérica (EN 0-10), escala facial (EF) y escala verbal análoga (EVERA) (Tabla 1). Para fines de certificación en el HRAEB, un grupo de expertos decidió tomar en cuenta a las poblaciones especiales que no pueden responder por sí mismas a una evaluación e incluyó las siguientes escalas: para pacientes sedados se aplicó la escala BPS (Behavioral Pain Scale); para neonatos se aplicó la escala CRIES (Crying, Requires oxygen SO2 > 95\%, Increased vital signs, Expression facial, Sleeplessness); y en niños que no verbalizan o en quienes no es suficiente la escala facial por su capacidad cognitiva, se aplicó la escala FLACC (Face, Leg, Activity, Cry, Consolability) $)^{13,14}$.

\section{Intervenciones previas a las mediciones}

Se diseñaron cinco acciones enfocadas hacia la atención del dolor en el paciente hospitalizado: 1) sensibilización del personal en el problema y el diagnóstico situacional; 2) actualización en analgesia multimodal por un grupo de anestesiólogos; 3) rediseño del formato de registro clínico del dolor en hospitalización, por parte de enfermería, anexando la gráfica correspondiente a la 5. a constante vital (dolor); 4) también por personal de enfermería, elaboración de un díptico titulado «Prevención y atención oportuna del dolor», que se distribuyó a todos los profesionales de enfermería del hospital, y 5) distribución de la escala del HRAEB para evaluar el dolor agudo entre el personal involucrado (áreas de Enfermería, Medicina y Anestesiología/ Algología).

\section{Diseño y población de estudio}

De acuerdo a las estadísticas del HRAEB en años previos, el mes de julio es representativo en cuanto a productividad quirúrgica, con aproximadamente 90 a 110 casos por semana, por lo que la estrategia fue revisar expedientes hasta alcanzar 25 casos incluidos en cada una de las semanas del mes. Al detectar la ausencia de una herramienta estandarizada para evaluar el dolor en el área médica (0\% de 100 expedientes evaluados) se dio inicio a las intervenciones. Se realizó un total de cuatro muestras de pacientes con dolor agudo posquirúrgico como fuente principal de dolor agudo en el HRAEB. Las primeras tres muestras, de 100 pacientes cada una, se hicieron con la intención de analizar el apego a la escala de la evaluación del dolor. La muestra 1 se realizó en julio de 2017, la muestra 2 en diciembre de 2017 y la muestra 3 en julio de 2018. Los pacientes se eligieron aleatoriamente y según su número de expediente registrado en la programación quirúrgica. Se determinó si hubo evaluación del dolor y si se había usado alguna escala para su cuantificación. Se excluyeron los pacientes quirúrgicos ambulatorios y aquellos que por alguna complicación perioperatoria se trasladaron a cuidados intensivos.

La muestra 4, realizada en el periodo de marzo a mayo de 2018, incluyó a 46 pacientes. Se aplicaron cuestionarios directos para determinar alivio del dolor postoperatorio con las intervenciones, el tipo de analgesia utilizada y la satisfacción del usuario con la experiencia en la atención específicamente del dolor. Se consideró un periodo máximo de 36 horas del periodo postoperatorio para la aplicación de los cuestionarios; se excluyeron seis cuestionarios por presentar datos incompletos.

\section{Definiciones}

\section{Variable 1}

Apego a la escala del HRAEB de evaluación del dolor agudo por el personal de salud.

- Apego a la escala por el área médica. Uso de la escala HRAEB en la primera nota de ingreso a hospitalización después de la cirugía. Tipo de variable: dicotómica nominal (Sí/No).

- Apego a la escala por el área de enfermería. Registro de la evaluación del dolor en escala numérica en la gráfica de las constantes vitales de la hoja de 
Tabla 2. Apego de evaluación del dolor agudo

\begin{tabular}{lccc}
\hline Datos & $\begin{array}{c}\text { Muestra 1 } \\
\text { julio 2017 }\end{array}$ & $\begin{array}{c}\text { Muestra 2 } \\
\text { diciembre 2017 }\end{array}$ & $\begin{array}{c}\text { Muestra 3 } \\
\text { julio 2018 }\end{array}$ \\
\hline Relación de sexos & $1: 1$ & $1: 1$ & $1: 1$ \\
Rango de edad & $1-78$ años & $1-81$ años & $5-79$ años \\
Evaluación en Enfermería & $10 \%$ & $33 \%$ & $68 \%$ \\
Evaluación por médicos & $0 \%$ & $35 \%$ & $48 \%$ \\
\hline
\end{tabular}

Fuente: Expediente electrónico y registro de cuidados enfermeros HRAEB.

cuidados enfermeros de hospitalización. Tipo de variable: dicotómica nominal (Sí/No).

\section{Variable 2}

Satisfacción del usuario con la aplicación del proceso de evaluación y atención al dolor posquirúrgico. Para medir el impacto del proceso de atención del dolor, se buscó la correlación estadística entre la atención del dolor posoperatorio y el nivel de satisfacción del usuario. Se utilizó como herramienta la adaptación de la aplicación del cuestionario de la APS para evaluación de calidad de la atención ${ }^{15}$. Cabe mencionar que, para fines de conclusión de esta auditoría, solo se analizó la correlación de las respuestas de cuatro preguntas adaptadas para este fin.

- Satisfacción del usuario en atención al dolor. Se refiere a la experiencia del usuario respecto al alivio del dolor postoperatorio y la atención recibida por todo el equipo tratante.

- Ejemplo pregunta: Indique en la siguiente escala lo que mejor exprese su satisfacción con la atención del dolor recibida en las últimas 24 horas. Tipo de variable: politómica nominal (excelente, muy buena, buena, suficiente y mala).

- Ejemplo pregunta: Indique en la siguiente escala lo que mejor exprese el alivio que ha experimentado con la atención del dolor recibida en las últimas 24 horas. Tipo de variable: politómica nominal (excelente, muy buena, buena, suficiente y mala).

- Expectativa del paciente en la atención del dolor postoperatorio.

- Ejemplo pregunta: Indique en la siguiente escala lo que mejor exprese el dolor que usted esperaba experimentar posterior a su cirugía. Tipo de variable: nominal dicotómica $(0-1,2-3,4-5,6,>6$ o dolor severo).

- Dolor postoperatorio experimentado por el paciente en las primeras 24 horas.

- Ejemplo pregunta: Indique en la siguiente escala lo que mejor exprese el dolor que ha experimentado posterior a su cirugía. Tipo de variable: nominal dicotómica (0-1, 2-3, 4-5, 6, > 6 o dolor severo).

\section{RESULTADOS}

En la tabla 2 se describen las muestras 1,2 y 3 , de 100 casos en cada una, con relación al apego del uso de la escala del HRAEB.

La muestra 1 se recabó en el mes de julio, el mes más representativo en cuanto a productividad quirúrgica; la muestra 2 se recabó al finalizar el primer año de actividades de la auditoría; la muestra 3 representa el mantenimiento del apego una vez concluida la sensibilización y capacitación del personal. El análisis estadístico con respecto al apego al estándar de evaluación del dolor del personal de salud se realizó mediante la prueba de chi-cuadrada $\left(\chi^{2}\right)$ (Fig. 1). El valor de $p$ fue significativo en ambas áreas $(p<0.001)$.

En la muestra 4 se aplicó el cuestionario de satisfacción mediante la herramienta de la APS con enfoque en las expectativas del usuario y su experiencia respecto a la atención del dolor agudo. Fueron en total 46 casos: se excluyeron 6 por datos incompletos en las respuestas; 22 fueron hombres y 18 mujeres en un rango de edad de 20 a 74 años. El $45 \%$ de los casos correspondió a cirugía oncológica, predominando el abdomen como sitio quirúrgico. En 27 de estos casos 
Cumplimiento evaluación del dolor estandarizada

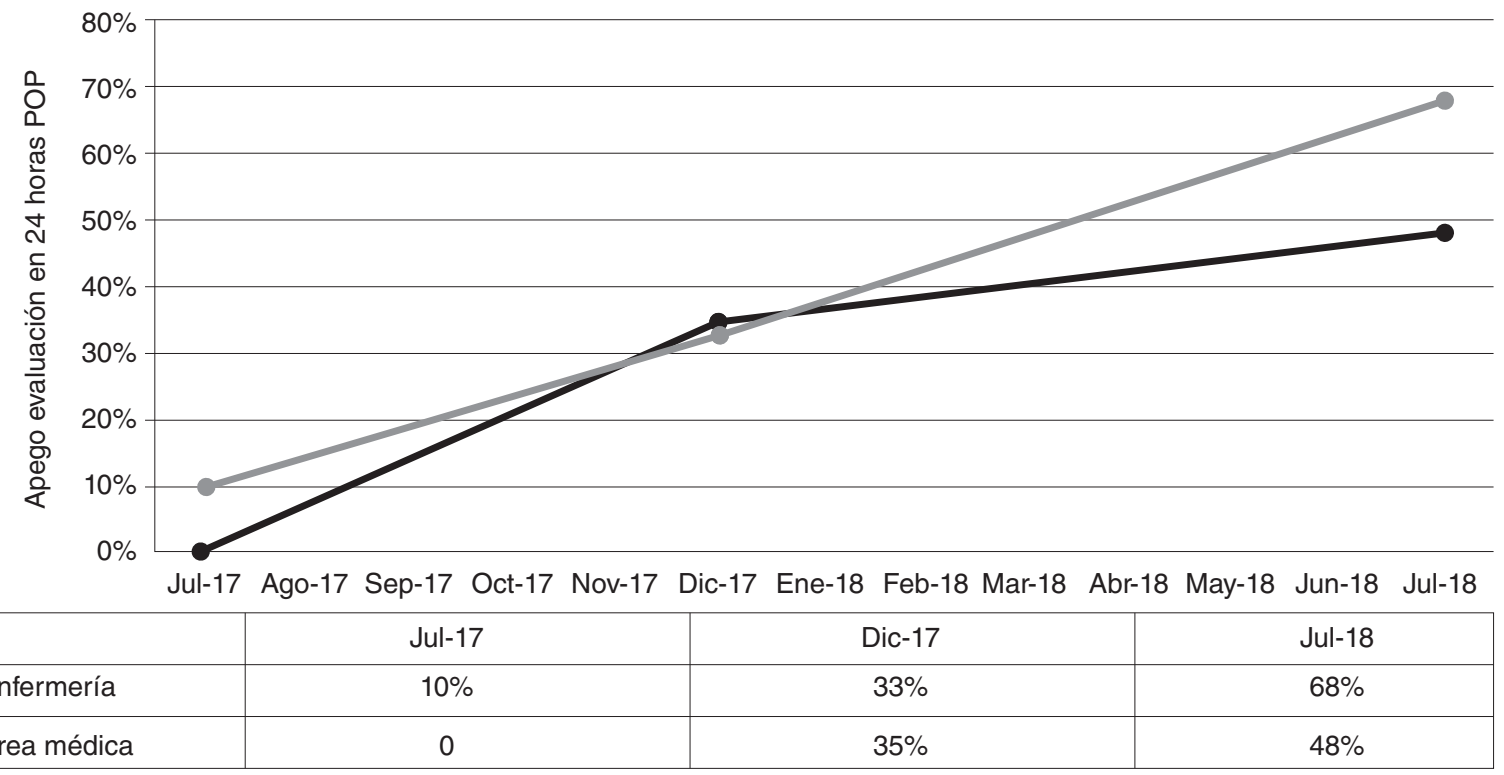

Análisis estadístico

\begin{tabular}{|c|c|c|c|c|c|c|}
\multicolumn{2}{c}{ Jul-17 } & Dic-17 & Jul-18 & X2 & X2 \\
\hline \multirow{2}{*}{ Enfermería } & 10 & 33 & 68 & 71.92 & $<0.001$ & 19.125 \\
\cline { 2 - 7 } & 90 & 67 & 32 & & & \\
\hline \multirow{2}{*}{ Área médica } & 0 & 35 & 48 & 57.37 & $<0.001$ & Infinito \\
\cline { 2 - 7 } & 100 & 65 & 52 & & & \\
\hline
\end{tabular}

Figura 1. Tendencia en el apego a la evaluación del dolor estandarizada, julio 2017-julio 2018. POP: postoperatorio; OR: odds ratio.

se utilizó analgesia multimodal, es decir, técnicas de anestesia regional además de mono o duoterapia de analgesia endovenosa.

En la figura 2 se muestra la distribución de los casos, con relación al tratamiento del dolor respecto a la escala numérica, sus expectativas y lo que experimentó el paciente posterior a la cirugía. Se aplicaron 46 cuestionarios basados en la versión en español de la APS, se descartaron 6 por información incompleta. De los 40 cuestionarios y con respecto a las cuatro mediciones específicas para satisfacción, 35 de los pacientes (87.5\%) esperaban que el dolor asociado a la cirugía fuera severo o mayor de 6 puntos en la escala numérica, aunque la percepción real del dolor evaluado en 5 rangos de la escala numérica y posterior a la cirugía fue menor a 4 puntos en el $92.5 \%$ de los encuestados. El análisis estadístico de ambas demuestra una correlación positiva, con un valor mediante la prueba de $\chi^{2}$ de 38.39 y una $p<0.001$ (significativa), así como un valor de la corrección $\mathrm{V}$ de Cramer de 0.8594.
En la figura 3 se representa el conjunto de las respuestas a los cuestionamientos del alivio del dolor con el esquema de analgésico utilizado y cómo percibió la atención del equipo tratante, incluyendo enfermería, médicos en hospitalización y la unidad de dolor agudo en caso de haber intervenido. La escala de ambas preguntas fue nominal y cualitativa, desde excelente hasta mala. Respecto al alivio del dolor postoperatorio, 33 pacientes $(82.5 \%)$ lo calificaron como excelente o muy bueno, correlacionando con la respuesta a dosis de rescate una vez que se solicitaba por el usuario en menos de 30 minutos. En 38 de 40 cuestionarios se registró como excelente-muy buena la atención brindada por el equipo tratante respecto al dolor. El análisis de correlación de rangos marcó un valor $\mathrm{R}$ de 0.948 , lo que implica una correlación directa del 94\%.

\section{Análisis de la muestra 4}

La percepción del paciente respecto a la atención del dolor dentro de un hospital suele ser negativa, 


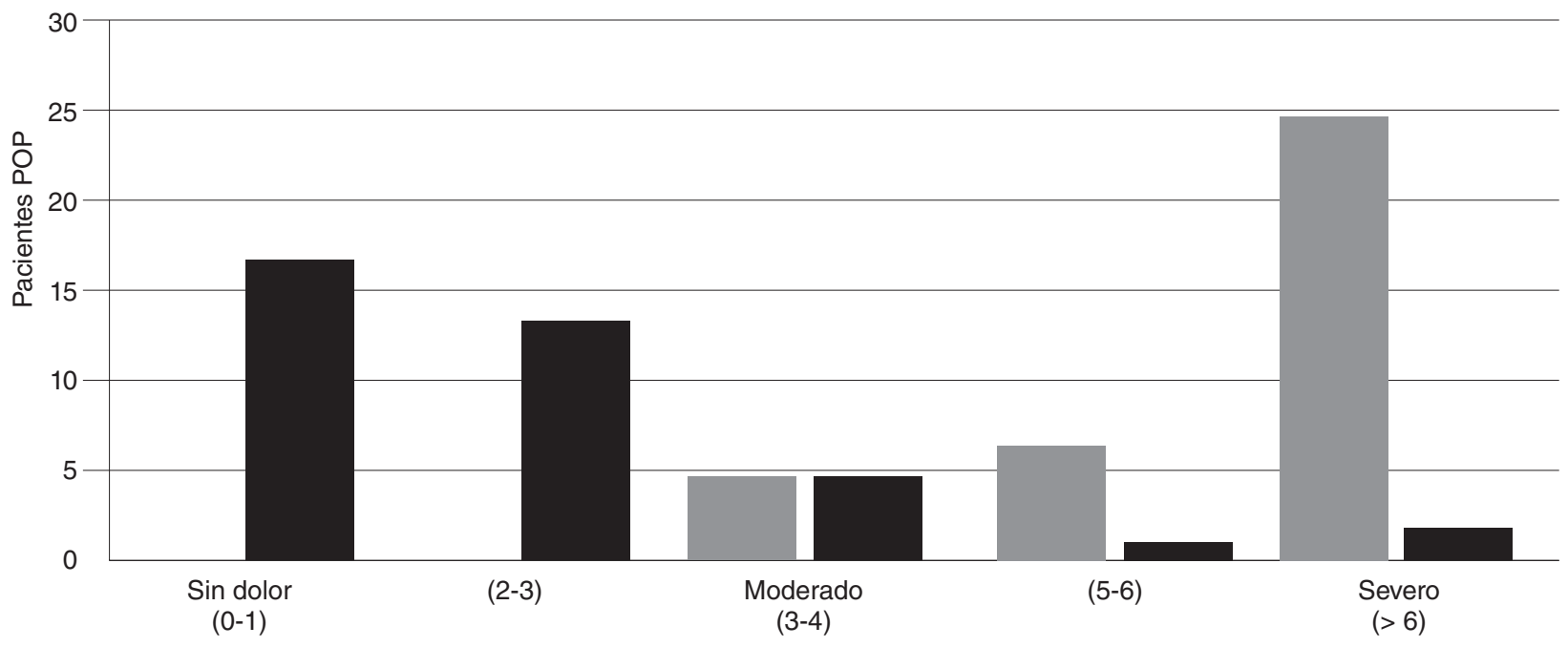

Clasificación equivalente a EN

Expectativa del usuario Dolor POP percibido

Respuestas a las preguntas en escala numérica de 5 rangos $(0-1,2-3,3-4,5-6$ y > 6); $n=40 ; p<0.001$.

Expectativa: «Indique en la siguiente escala lo que mejor exprese el dolor que usted esperaba experimentar posterior a su cirugía.» Dolor percibido: «Indique en la siguiente escala lo que mejor exprese el dolor que ha experimentado posterior a su cirugía.»

Figura 2. Correlación de alivio/expectativas del paciente sometido a cirugía y el dolor experimentado.

Percepción de la atención

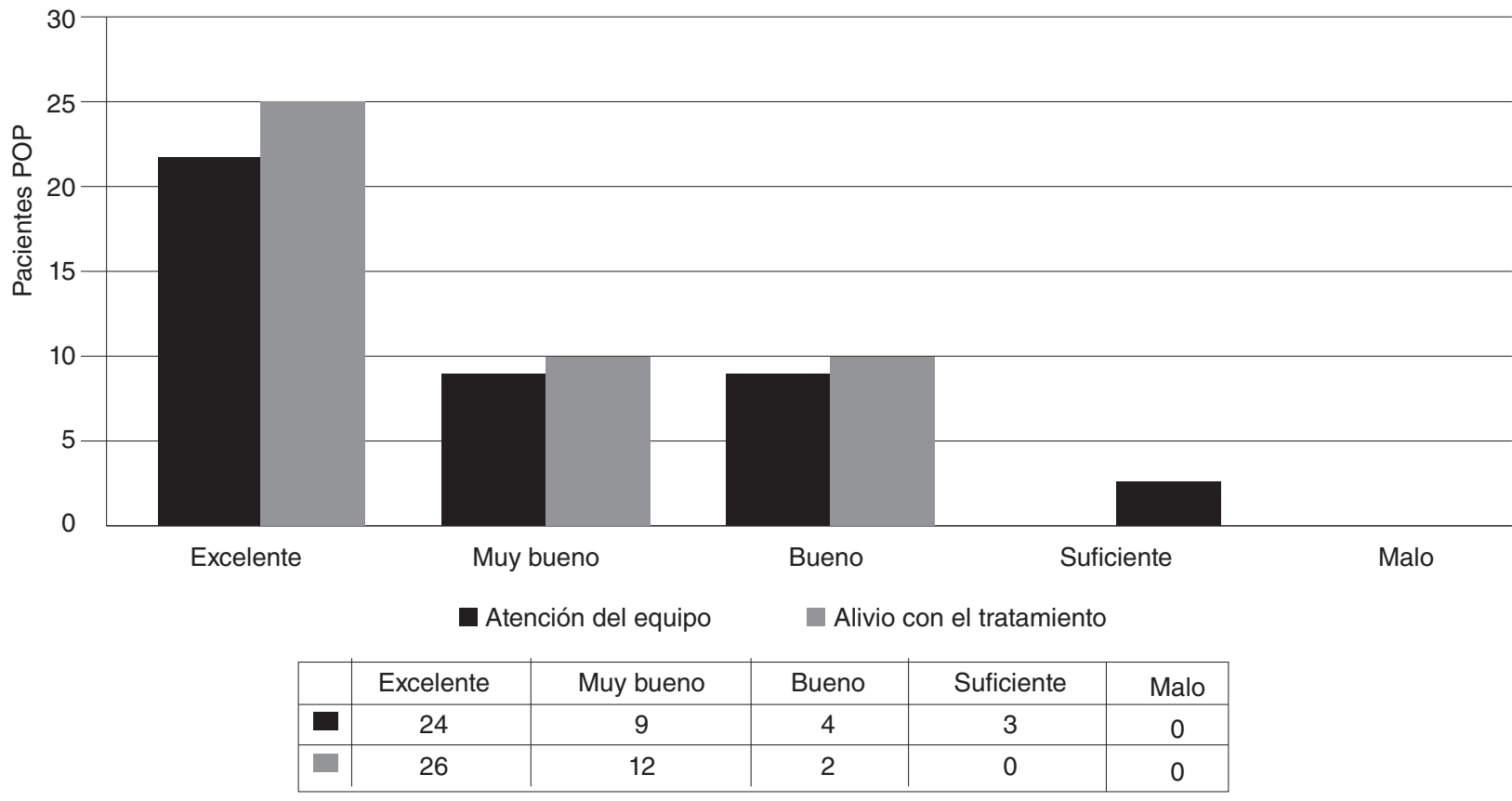

Respuesta a las siguientes preguntas en escala nominal en 5 rangos: excelente, muy bueno, bueno, suficiente y malo; $n=40$; correlación R: 0.94; correlación directa en 94\%.

Atención: «Indique en la siguiente escala lo que mejor exprese su satisfacción con la atención del dolor recibida en la últimas 24 h.» Alivio percibido: «Indique en la siguiente escala lo que mejor exprese el alivio que ha experimentado con la atención del dolor recibida en la últimas 24 h.»

Abreviaturas. POP: postoperatorio.

Figura 3. Correlación del alivio del dolor con la percepción del usuario de la atención. 


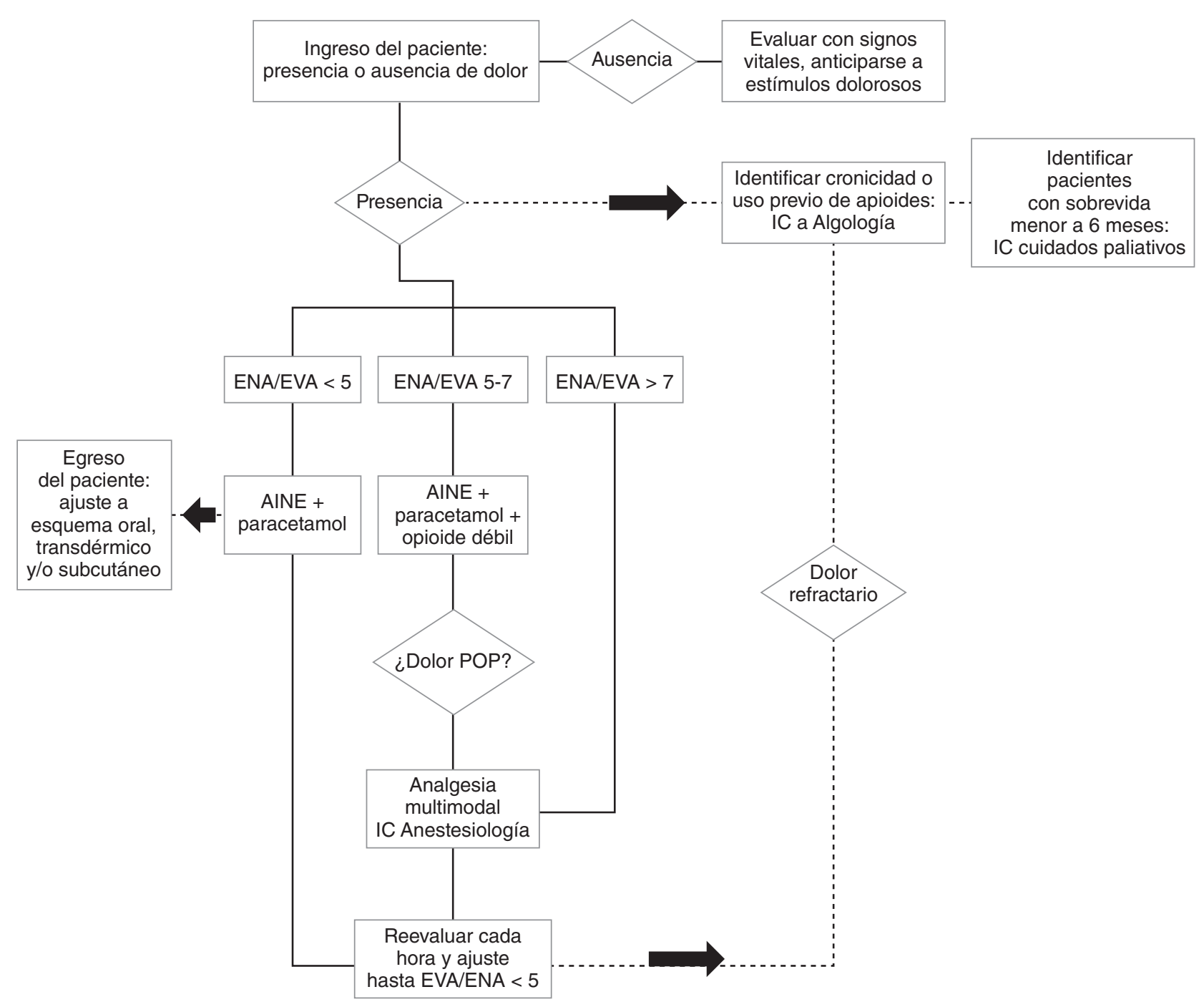

Figura 4. Algoritmo del proceso de atención al dolor en el Hospital Regional de Alta Especialidad del Bajío (HRAEB). Fuente: proceso COP (care of patients) 8 HRAEB.

considerando que 35 de 40 pacientes contestaron que esperaban tener dolor severo después de su cirugía. Cuando se les preguntó sobre dolor percibido dentro de las primeras 24 horas posteriores a la cirugía (en la misma escala en que se cuestionaban sus expectativas), el $37 \%$ lo registró como leve a moderado, en escala numérica menos de 5 puntos (que se había establecido como meta durante las intervenciones de dolor agudo, ver Fig. 4). De manera adicional, se consultó el registro de farmacia del consumo promedio mensual (CPM) de las diferentes presentaciones de opioides durante 2017 y primer semestre de 2018, comparado con 2016, con el objetivo de observar algún incremento o descenso fuera de la tendencia previa. Los resultados se observan en la figura 5; los cambios porcentuales son menores al crecimiento anual proyectado para la atención hospitalaria (9\%), incluso para el propio Servicio de Anestesiología (11\%).

\section{DISCUSIÓN}

En 2017, los pacientes posquirúrgicos en el HRAEB representaron el $79 \%$ de los egresos hospitalarios, esto es, 5,533 pacientes. Todos ellos fueron evaluados en las dos unidades de cuidados postanestésicos del hospital y se inició el esquema de analgesia de acuerdo al grado de estímulo doloroso (cirugía abierta o de mínima invasión) y al sitio quirúrgico. En la unidad de cuidados postanestésicos, el 96\% fueron evaluados de acuerdo con las escalas estandarizadas 


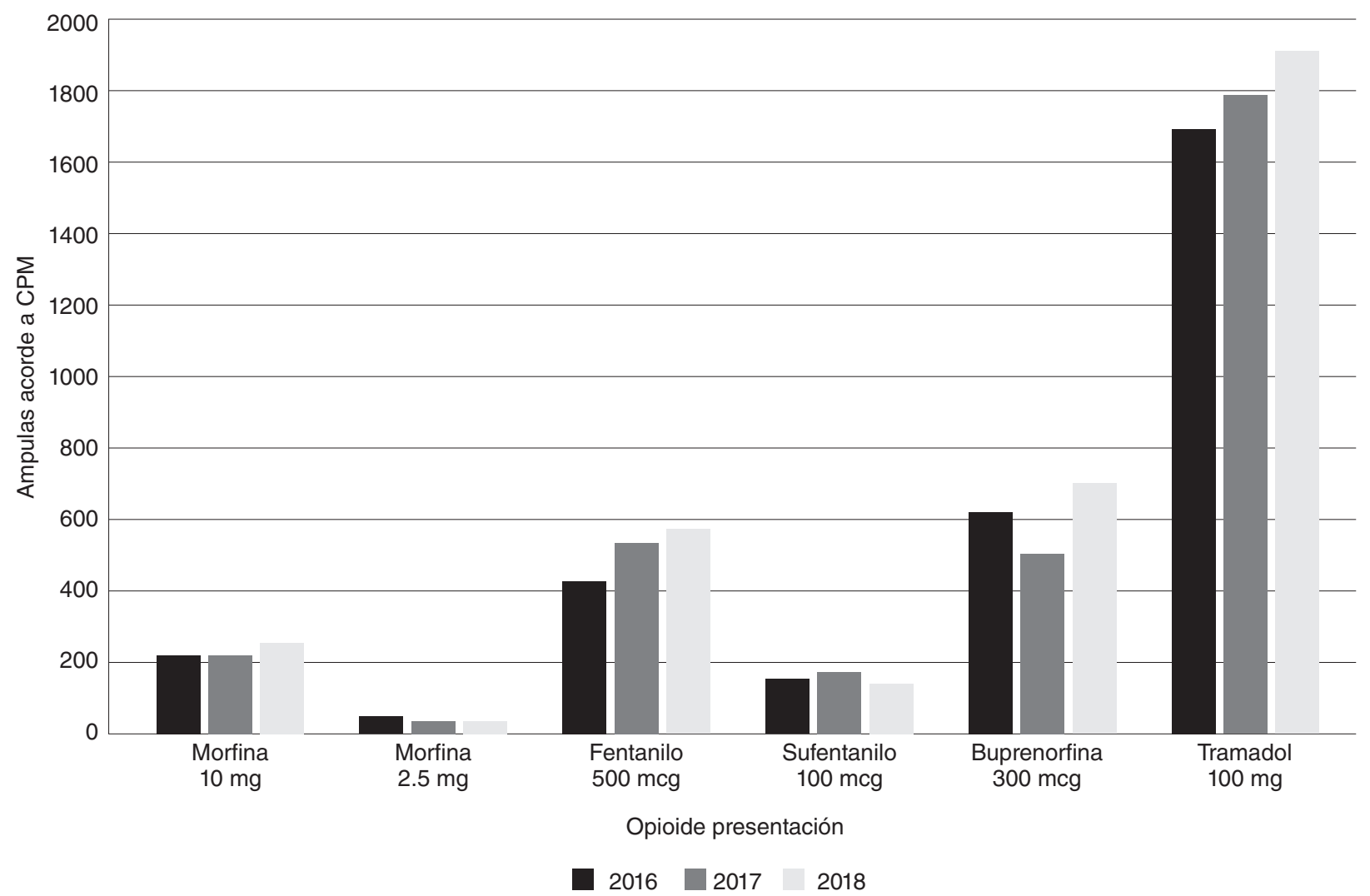

Diferencia porcentual 2016-2017: morfina $10 \mathrm{mg}, 2.5 \mathrm{mg}$ y buprenorfina $300 \mu \mathrm{g}$, negativos. Fentanilo $500 \mu \mathrm{g}, 20 \%$;sufentanilo $100 \mu \mathrm{g}$, 16\%; y tramadol $100 \mathrm{mg}, 5 \%$. En 2018 disminuye el consumo de sufentanilo y la diferencia de fentanilo baja a $6.5 \%$.

Figura 5. Consumo de opioides en el 2017 de acuerdo al registro de consumo promedio mensual (CPM). Fuente: Registro CPM de Farmacia en Kewan HRAEB.

por los anestesiólogos y si la puntuación durante los cuidados de enfermería era equivalente a más de 5 o severo, el personal de enfermería del área solicitaba intervención; el 4\% restante corresponde a los pacientes que fueron trasladados directamente de quirófano a otras áreas. Cabe mencionar que durante las capacitaciones de enfermería se hizo énfasis en las bondades de la analgesia multimodal, que incluía la permanencia de un catéter epidural en el postoperatorio. El apego de evaluación y registro del dolor se midió una vez que el paciente era admitido en hospitalización, con notas médicas del curso clínico y en la hoja de cuidados enfermeros. El apego fue en aumento conforme las actividades de la auditoría progresaron, en buena parte por la propia convicción del personal involucrado de repercutir positivamente en la experiencia del paciente en hospitalización, e incluso en la percepción del cuidador. Sin duda, el personal involucrado en la atención del dolor agudo tuvo buen recibimiento y mayor apego al uso de las escalas establecidas en el proceso a la par que la auditoría avanzaba en la estrategia de dar a conocer las buenas prácticas del proceso. El área médica se sintió muy cómoda con la escala unificada y partió de no evaluar con alguna escala (se utilizaban términos como «dolor bien tolerado», «dolor presente» o «dolor esperado para la cirugía») al 48\% de cumplimiento con la escala HRAEB (Fig. 1). Esto permitió que pudiera atenderse con mayor efectividad el dolor; al registrar puntuaciones mayores a 5 se solicitaba la atención, ya fuera indicada como dosis de rescate o como revaluación por el médico tratante. En cuanto al área de enfermería, fueron protagonistas en anticiparse al dolor, teniendo una correlación directa en los pacientes encuestados para alivio del dolor (37 de 40) con puntuaciones menores a 5. De igual forma, los Servicios de anestesiología y cirugía trabajaron en conjunto y con base en las buenas prácticas para diseñar de manera coordinada la analgesia, de tal manera que antes de la cirugía o antes de que iniciara el dolor se tenía la pauta de acción que sería la prescripción médica. 
Por otro lado, es importante reconocer que, lejos de consumir más opioides en el alivio del dolor, se puede interpretar que los incrementos en el CPM reportados en estos años están por debajo de la diferencia porcentual del crecimiento de población atendida en el HRAEB. EI consumo de la buprenorfina pudiera ser la excepción, aunque, al analizar las salidas, estas se registraron para consumo de las unidades de cuidados intensivos, situación que también pudiera reflejar el uso de escalas de evaluación de dolor en pacientes sedados. Es prematuro concluir que los opioides no fueron necesarios durante la realización de la auditoría clínica, mejor dicho, no fueron la solución al problema del dolor. Insistimos en que la prevención permitió tratar el dolor desde puntuaciones muy bajas con esquemas que usan opioides por menos de 24 horas o incluso prescinden de ellos.

Finalmente, se debe reconocer que existen resultados tangibles de esta auditoría. Por ejemplo, se elaboró el manual del proceso de atención de dolor que, además de vincular los servicios clínicos involucrados, formó parte de la certificación hospitalaria en el estándar AOP y COP ${ }^{11}$. El funcionamiento de la unidad de dolor agudo fue de bajo costo, es decir, no requirió de más recursos, sino solo la estructuración de los ya presentes, además del inestimable apoyo del personal en formación de Anestesiología, Cirugía y Enfermería. El apego y actualización en buenas prácticas de todo el personal ha sido permanente posterior a las capacitaciones, incluso ya con propuestas nuevas. Dentro de estas, por ejemplo, está el apoyar la terapéutica del dolor no farmacológica por parte de Enfermería, el diseño de nuevas escalas multidimensionales para evaluación del dolor y el proyecto del procedimiento para atención perioperatoria del paciente de cuidados paliativos.

Sin duda alguna, la realización de esta auditoría clínica contribuyó a percibir la necesidad de contar con analgesia multimodal, técnicas focalizadas guiadas por ultrasonido, anestésicos locales previos a las incisiones o colocación de puertos para tener usuarios más satisfechos y servicios médicos de calidad centrados en el paciente.

\section{CONCLUSIONES}

La auditoría clínica en atención del dolor agudo durante estos dos años ha representado una herramienta que permitió:
- Identificar procesos incompletos (atención del dolor sin vincular a otros servicios clínicos relacionados en ello).

- Obtener variables medibles para mejora (no se disponía de una escala estandarizada para evaluar y poder dar seguimiento al apego del proceso).

- Obtener variables de calidad en atención clínica (convertir la atención del dolor en una variable de calidad en el paciente hospitalizado).

- Vinculación entre servicios clínicos y de calidad (el proceso como proyecto del área de Calidad y Anestesiología).

- Cumplir con estándares de certificación.

- Mayor satisfacción del usuario en su experiencia durante la hospitalización asociada a eventos quirúrgicos.

\section{SEGUIMIENTO DE LA AUDITORÍA}

Sin duda, será necesario abarcar toda la población atendida en el hospital en cuanto a dolor se refiere. Convertir la auditoría en un comité clínico permanente representa la oportunidad de integrar el resto de los servicios hospitalarios. Se deberá monitorizar permanentemente el consumo de los opioides disponibles en el HRAEB, así como favorecer las políticas necesarias que otorguen seguridad y calidad en su prescripción; esto representa el compromiso de participar activamente en disminuir un problema de salud pública como es el dolor crónico. Esta auditoría clínica es el ejemplo de una organización convencida del beneficio de apegarse a un procedimiento. El estándar próximo por alcanzar es el $100 \%$ de apego por ambas áreas.

\section{BIBLIOGRAFÍA}

1. Max MB. Improving outcomes of analgesic treatment: is education enough? Ann Intern Med. 1990;113(11):885-9.

2. McQuay H, Moore A, Justins D. Treating acute pain in hospital. BMJ. 1997;314:1531-5.

3. American Pain Society Quality of Care Committee. Quality improvement guidelines for the treatment of acute pain and cancer pain. JAMA. 1995;274: 187-80.

4. Gan TJ. Poorly controlled postoperative pain: prevalence, consequences, and prevention. J Pain Res. 2017;10:2287-98. 
5. Brennan F, Carr DB, Cousins MJ. Pain management: a fundamental human right. Anesthesia Analgesia. 2007;105:205-21.

6. Baker DW. History of The Joint Commission's Pain Standards Lessons for Today's Prescription Opioid Epidemic. JAMA. 2017;317(11):1117-8.

7. Cregg R, Anwar S, Farquhar-Smith P. Persistent postsurgical pain. Curr Opin Support Palliat Care. 2013;7:144-52.

8. Erazo MA, Pérez L, Colmenares CC, Álvarez H, Suárez I, Mendivelso F. Prevalencia y caracterización del dolor en pacientes hospitalizados. Rev Soc Esp Dolor. 2015;22(6):241-8.

9. Covarrubias A. El manejo del dolor agudo postoperatorio: Una década de experiencias. Rev Mex de Anest. 2013;36(1):S179-82.

10 Baker DW. History of The Joint Commission's Pain Standards: Origins and Evolution. Oakbrook Terrace, IL: The Joint Commission; 2017.

11. Modelo de Seguridad del paciente del Sistema Nacional de Certificación de Establecimientos de Atención Médica. Estándares para implementar el modelo en Hospitales. México: Secretaría de Salud; 2018.
12. Chou R, Gordon DB, de Leon-Casasola OA, Rosenberg JM, Bickler S, Brennan T, et al. Management of Postoperative Pain: A Clinical Practice Guideline From the American Pain Society, the American Society of Regional Anesthesia and Pain Medicine, and the American Society of Anesthesiologists' Committee on Regional Anesthesia, Executive Committee, and Administrative Council. J Pain. 2016;17(2):131-57.

13. Bendinger T, Plunkett N. Measurement in pain medicine. Br J Anaesth Education. 2016;16(9):310-5.

14. Suraseranivongse $S$, Kaosaard R, Intakong P, Pornsiriprasert $S$, Karnchana $Y$, Kaopinpruck J, et al. A comparison of postoperative pain scales in neonates. Br J Anaesth. 2006;97(4):540-4.

15. Gordon DB, Polomano RC, Pellino TA, Turk DC, McCracken LM, Sherwood G, et al. Revised American Pain Society Patient Outcome Questionnarie (APS-POQ-R) for quality improvement of pain management in hospitalized adults: preliminary psychometric evaluation. 2010;11(11): 1172-86. 